\title{
Mitral Valve Reconstruction with Artificial Chordae: How to Secure the Desired Length?
}

\author{
M Krane ${ }^{1}$, EU Braun ${ }^{1}$, H Mayer $^{2}$, A Knoll $^{2}$, \\ R Bauernschmitt ${ }^{1}$, R Lange ${ }^{1}$ \\ ${ }^{1}$ German Heart Center Munich, Munich, Germany \\ ${ }^{2}$ Technical University Munich, Munich, Germany
}

\begin{abstract}
The use of sutures as artificial chordae is widely established in mitral valve reconstruction. After the correct length of the chordae is determined by saline injection, there is a variety of methods to secure that length before tying surgical knots. We investigated the amount of damage posed by applying a haemoclip or a pean clamp and by using a knot-pusher. No differences in breaking forces were found, if a pean clamp was applied, with or without the use of a knot-pusher. (control group: $36 \pm 4.4 \mathrm{~N}$; Pean: $37.5 \pm 4.7 \mathrm{~N}$; control group + knotpusher: $38.6 \pm 5 N$; pean + knot-pusher: $37.5 \pm 4.2 N$ ). Using a haemoclip significantly decreased the breaking forces to $12.9 \pm 14.6 \mathrm{~N}$ compared to control group $(p<0.01)$. The length can be safely secured by applying a pean clamp. Haemoclips should not be used on artificial chordae.
\end{abstract}

\section{Introduction}

Mitral valve repair became the first surgical option in patients with degenarativ mitral valve disease. Different techniques for mitral valve repair were developed during the last decades. One established technique for mitral valve repair with good long term results [1] is the placement of artificial chordae using polytetrafluoroethylene (PTFE) sutures (GORE-TEX ${ }^{\circledR}$ Suture CV-4, W. L. Gore \& Associates, Inc., Flagstaff/ AZ, USA). Once the desired length of the suture is determined the surgeon has to tie the sliding knot. Different techniques were used to prevent the sliding of the knot. We routinely use a pean clamp. During minimal invasive antero-lateral thoracotomy for mitral valve repair a knot-pusher has to be used for tying surgical knots. In this study we investigated the breaking strength of Gore-Tex sutures which were clamped by a pean clamp or a haemoclip compared to unclamped sutures while tying surgical knots.

\section{Methods}

\section{Experimental setup}

The thread of the surgical suture material was GORETEX $^{\circledR}$ Suture CV-4 (W. L. Gore \& Associates, Inc., Flagstaff/ AZ, USA), which was clamped between two robot arms (Mitsubishi MELFA RV-6SL, Mitsubishi Electric Corporation, Tokyo, Japan) (Figure $1+2$ ).

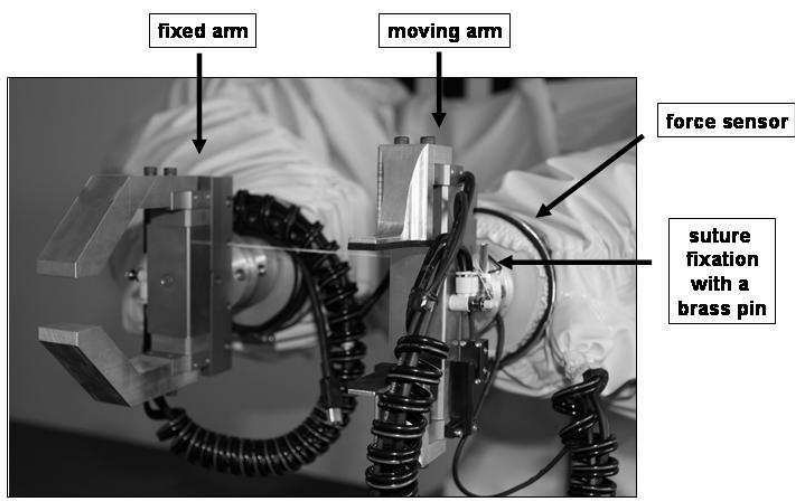

Figure 1: The tied suture clamped between the two robot arms (Mitsubishi MELFA RV-6SL, Mitsubishi Electric Corporation, Tokyo, Japan).

The velocity of traction of the suture material was $5 \mathrm{~cm}$ per second (same velocity in every trial). One robot arm was equipped with a force sensor (Mini40, ATI Industrial Automation, Apex/ NC, USA) to measure the forces while pulling the suture in one direction $\left(\mathrm{F}_{\mathrm{x}}\right)$ (Figure 1, 2). The sample rate was 7 milliseconds. The force registration software (developed by the Institute of Robotics and Embedded Systems, Technische Universität München, Germany) recorded the force $F_{x}$. 


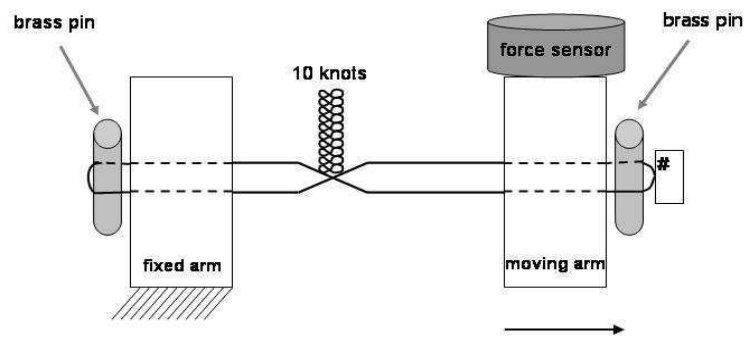

Figure 2: experimental setup with the fixed GORE-TEX ${ }^{\circledR}$ Suture between the two robot arms.

\section{Fixing of the suture material}

For the in vitro test the strength of suture material was evaluated by 2 experiments with different techniques to ensure the suture length before tying surgical knots.

First experiment: The first experiment consists of three groups. In the pean group, the desired length was secured by using a pean clamp. In the clip group a haemoclip (SLS haemostatic clip $^{\mathrm{TM}}$, small, Vitalitec Surgical, Vitalitec Inc./France) was used to fix the desired length of the suture material before surgical tying. In the control group no instrument or clip was applied to fix the length of the GORE-TEX ${ }^{\circledR}$ suture. After secure the desired length 10 knots were tied by the same surgeon. In experiment 1 all knots were tied by using a knot-pusher.

Second experiment: The second experiment consists of two grous. In the pean group and the control group 10 knots were tied by the same surgeon with bare fingers to evaluate the influence of the knot-pusher.

In both experiments spontaneous breaking of the suture was defined to be caused by a force of $1 \mathrm{~N}$ or less.

\section{Statistical analysis}

Results are presented as mean \pm 1 standard deviation. The t-Test was used to analysis differences between the groups. A p-value less or equal to 0.05 was regarded as significant. All statistical analyses were performed using SPSS 15 software (SPSS, Chicago, Illinois).

\section{Results}

The point of rupture of all sutures was located in both experiments at the knot (Figure 3).

\section{First experiment}

In the pean group the breaking force was $37.5 \pm 4.2 \mathrm{~N}$, in the clip group $12.9 \pm 14.6 \mathrm{~N}$ and in the control group $38.6 \pm 5 \mathrm{~N}$.

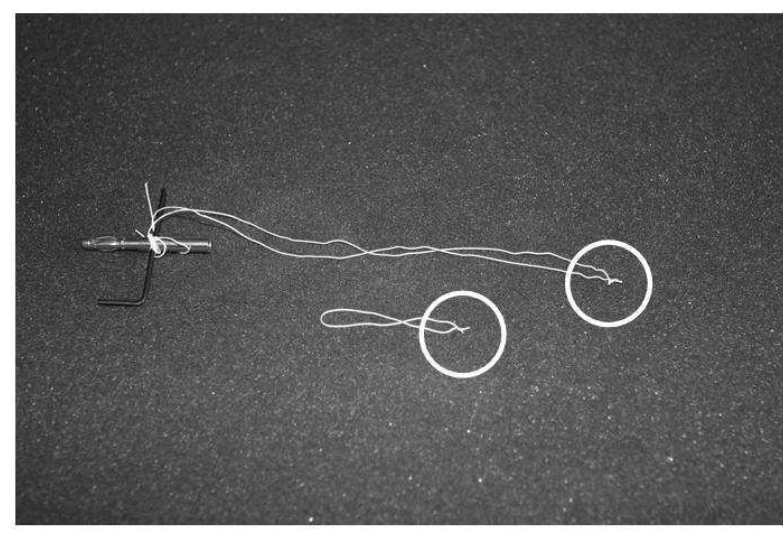

Figure 3: Ruptured suture. All sutures ruptured at the knot.
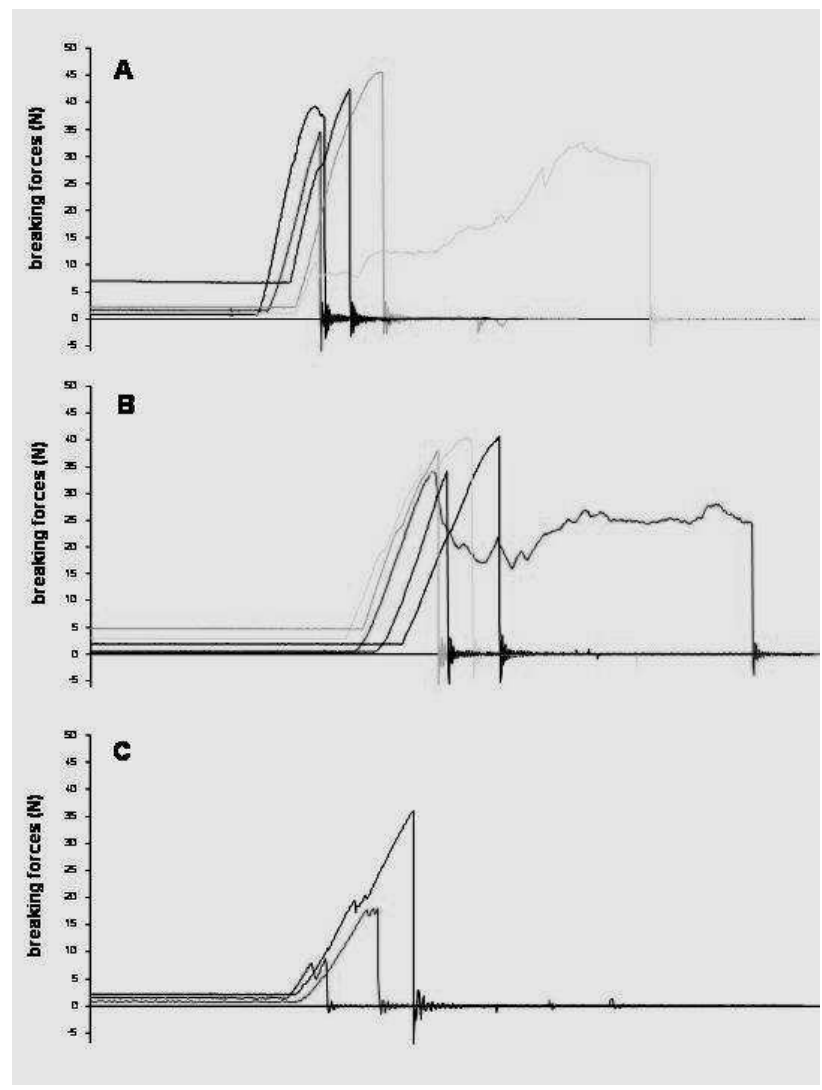

Figure 4: The single experiments of the pean group (A), the control group (B) and the clip group (C) in experiment 1 . All sutures in this series were tied using a knot-pusher.

The decreased breaking forces between the clip group 
compared to the pean group or the control group was statistically significant $(\mathrm{p}<0.01)$ (Figure 6). Two sutures in the clip group broke spontaneously. In the pean group and the control group no suture broke spontaneously.

\section{Second experiment}

In the pean group the breaking force was $37.5 \pm 4.7 \mathrm{~N}$ and in the control group $36 \pm 4.4 \mathrm{~N}$.

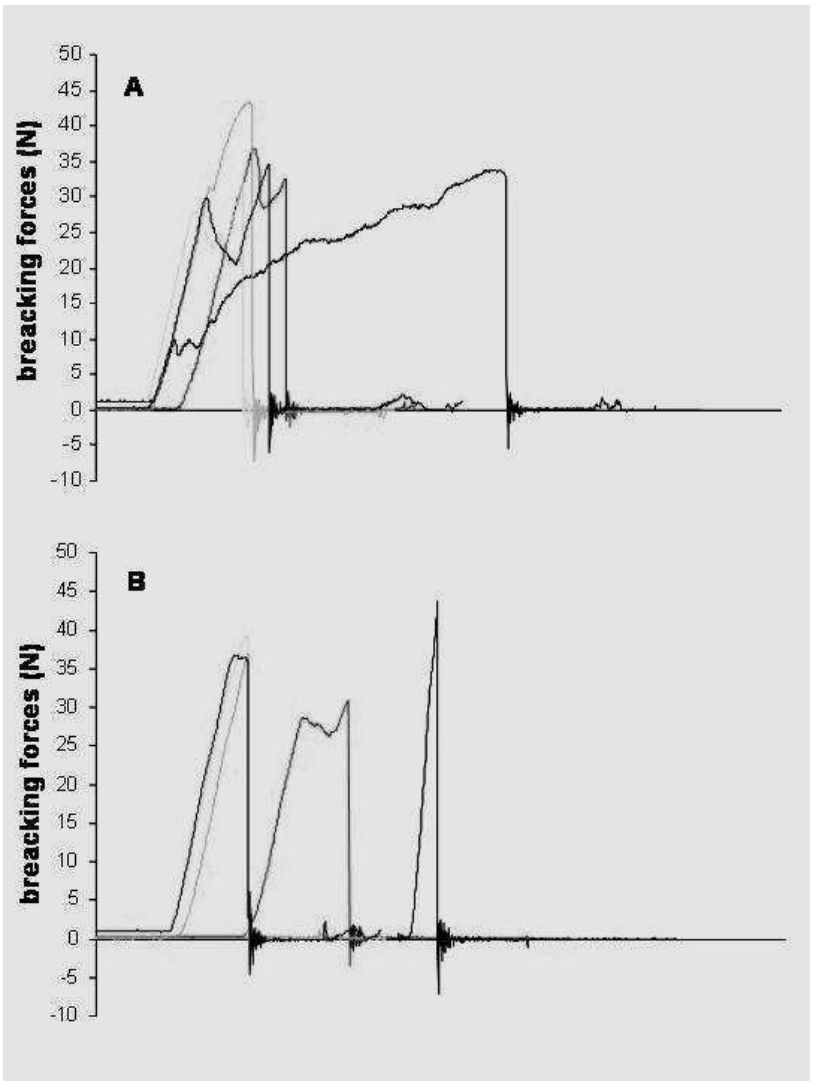

Figure 5: The single experiments of the pean group (A) and the control group (B) in experiment 2. All sutures in this series were tied without using a knot-pusher.

Comparing the results of the pean group of experiment 1 to the pean group of experiment 2 no differences were found. Comparing the results of the control group of experiment 1 to the control group of experiment 2 no differences were found (Figure 6).

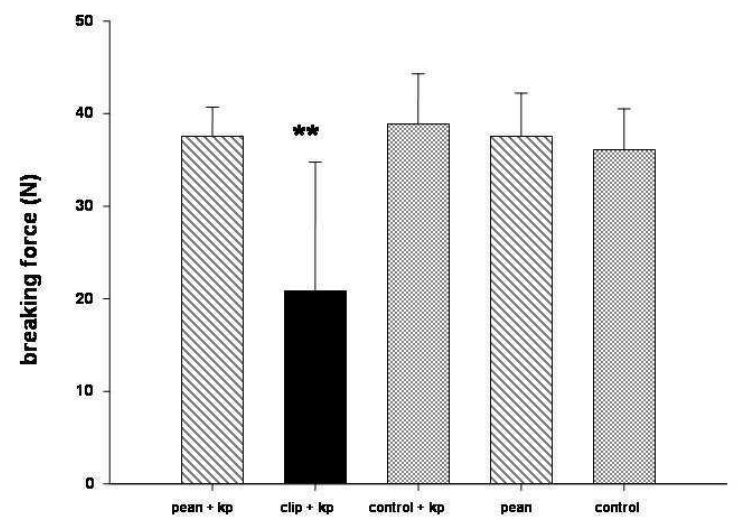

Figure 6: Breaking forces of the pean, clip and control group of both experiments (mean \pm standard deviation). $\mathrm{kp}=$ knot-pusher; $* *$ significantly decreased $\mathrm{p}<0.01$

\section{Discussion and conclusions}

No differences for breaking forces were found between the control group and the pean group. However, the breaking forces in the clip group were significantly decreased. Furthermore, no differences in breaking forces were found between sutures of the pean group and the control group tied with or without a knot-pusher.

In 1985, Gore-Tex sutures were introduced in mitral valve surgery. Today, placement of artificial chordae became a standard procedure for mitral valve repair with good long-term results [1]. The Gore-Tex sutures have a breaking strength superior to native chordae and maintain the flexibility similar to a physiological chordae [2]. Dang and co-workers [3] compared the biomechanical considerations of PTFE sutures to polypropylene sutures. The knot construction significantly reduced the breaking strength of polypropylene sutures but did not alter the breaking strength of PTFE sutures.

One of the biggest pitfalls in placement of artificial chordae is the adjustment of the correct length. It is very easy to tie the Gore-Tex suture down too far and provide a restricted portion of the "corrected" leaflet. Difficulties in secure the correct length of the artificial chordae and tying the suture without knot slipping lead to development of various techniques and complex new instruments [2; 4-6].

Matsui and co workers [4] described a new device for ensuring the desired length of the artificial chordae and provide tying without knot slipping. The new tool consists of a circular, hook-shaped distal tip and a proximal hook.

Oppell and Mohr [2] described a method using a 
vernier calliper device as a template to make a Gore-Tex loop of a premeasured length. This premeasured loop will be secured by knotting over a pledget. Thereafter, the pledget will be fixed at the papillary muscle and the opposite end of the loop will be fixed to the prolapsing leaflet.

We routinely use a pean clamp to secure the desired length of an artificial chordae. The Gore-Tex suture will be fixed at the papillary muscle tip. Thereafter both ends of the Gore-Tex suture will be fixed at the prolapsing leaflet. The correct height will be determined by saline injection. Afterwards a pean clamp will be used to secure the desired length and to avoid knot slipping during tying the Gore-Tex suture. In case of a lateral minithoracotomy a knot pusher have to be used for tying surgical knots. In most cases of median thoracotomy the use of a knot-pusher is not necessary. The advantage of our method is an ubiquitary available instrument which is easy to use. The use of an applied pean clamp did not lead to a decreased breaking strength due to a possible additional damage of the Gore-Tex suture. Furthermore, the use of a knot-pusher did not lead to decreased breaking forces, too.

The use of a haemoclip to secure the desired length of an artificial chordae leads to a highly increased risk for rupture of the Gore-Tex suture.

\section{References}

[1] David TE, Omran A, Armstrong S, Sun Z, Ivanov J. LongTerm results of mitral valve repair for myxomatous disease with and without chordal replacement with expanded polytetrafluoroethylen sutures. J Thorac Cardiovasc Surg 1998; 115: 1279-1286

[2] von Oppell UO, Mohr FW. Chordal replacement for both minimally invasive and conventional mitral valve surgery using premeasured Gore-Tex loops. Ann Thorac Surg 2000; 70: 2166-8

[3] Dang MC, Thacker JG, Hwang JC, Rodeheaver GT, Melton SM, Edlich RF. Some biomechanical considerations of polytetrafluoroethylene sutures. Arch Surg 1990; 125: 647-0

[4] Matsui Y, Fukada Y, Naito Y, Sasaki S, Yasuda K. A new device for ensuring the correct length of artificial chordae in mitral valvuloplasty. Ann Thorac Surg 2005; 79:1064-5

[5] Sarsam MAI. Simplified technique for determining the length of artificial chordae in mitral valve repair. Ann Thorac Surg 2002; 73: 1659-60

[6] Adams DH, Kadner A, Chen RH. Artificial mitral valve chordae replacement made simple. Ann Thorac Surg 2001; 71: 1377-9

Address for correspondence

Dr. Markus Krane

German Heart Center Munich

Clinic for cardiovascular surgery

Lazarettstr. 36

80636 Munich

krane@dhm.mhn.de 